\title{
ENFOQUE ERGONÓMICO/FACTORES HUMANOS PARA PROFESIONALES DE LA SALUD EN TIEMPOS DE PANDEMIA. A PROPÓSITO DE UNA EXPERIENCIA
}

\author{
ERGONOMIC/HUMAN FACTORS APPROACH FOR HEALTH PROFESSIONALS IN TIME \\ OF PANDEMIC. PURPOSE OF AN EXPERIENCE
}

Humberto Tapia-Escalante ${ }^{*}$

\begin{abstract}
Resumen: En tiempos de pandemia los sistemas de salud mostraron sus verdaderas debilidades, se generaron miles de contagios en las ciudades, como también en el personal sanitario. El enfoque ergonómico y factores humanos por ser de carácter proactivo, clave para mejorar los sistemas evitando así accidentes, eventos adversos y complicaciones que puedan afectar directamente la seguridad de los pacientes y de los trabajadores de salud, tomó significativa pertinencia. El objetivo de la presente experiencia fue incorporar en los sistemas de gestión de las empresas mediante el uso de medios digitales, tales como video consultas y encuestas digitales, la vigilancia de la salud. Se atendieron requerimientos de personas en aislamiento por COVID-19 a través de 300 video consultas, y de personal en riesgo, con 36.000 datos de encuestas de salud durante seis meses de seguimiento. En el grupo vigilado no se presentó ninguna complicación fatal. Esta práctica a pesar de las limitaciones técnicas, tecnológicas y económicas, su aplicación es factible, y optimizándola garantiza amplias coberturas, limitados contactos y contagios, y por otra parte, tiene amplias potencialidades para su aplicación no solo en tiempos de enfermedades transmisibles, sino en diversas eventualidades del ciclo salud-enfermedad.
\end{abstract}

Palabras clave: Ergonomía y factores humanos, tele-salud, videoconsultas, COVID-19.

\begin{abstract}
In times of pandemic, health systems showed their real weaknesses; thousands of contagions were generated in the cities and also, among the health personnel. The ergonomic approach and human factors for being proactive, the key to improving systems, thus avoiding accidents, adverse events, and complications that may directly affect patients and health workers' safety took on significant relevance. The objective of the present experience was to incorporate health surveillance in the management systems of the companies through the use of digital media, such as video consultations and digital surveys. The requirements of people in isolation by COVID-19 were met through 300 video consultations and personnel at risk, with 36,000 health survey data during six months of follow-up. No fatal complications occurred in the monitored group. Despite the technical, technological and economic limitations, this practice is feasible, and optimizing it guarantees wide coverage, limited contacts and contagions, and on the other hand, it has excellent potential for its application not only in times of infectious diseases but also in various eventualities of the healthdisease cycle.
\end{abstract}

*Centro de Estudios del Trabajo. Loja, Ecuador. Correo electrónico: humbertotapia2010@yahoo.com. Orcid: https://orcid.org/oooo-0oo3-3740-7237 
Keywords: Ergonomics and human factors, telehealth, video consultations, COVID-19.

Recepción: 15.06.2021 / Revisión: 29.06.2021 / Aceptación: 30.07.2021

\section{Introducción}

La forma exponencialmente de conectar al mundo marca un antes y un después en la historia de la humanidad a partir del internet y las tecnologías de la información y comunicación. Esta puerta se abrió para todas las actividades humanas y las ciencias, sus aplicaciones y/o usos imaginables e inimaginables; una de estas ciencias es la de la salud, donde se utilizan en la formación profesional, el intercambio de experiencias, para el apoyo remoto a equipos de profesionales de ciencias biomédicas, para la prestacion de servicios, etc. Además, también se hace uso de ellas en el ámbito privado, y a la fecha en importante medida en las entidades públicas.

Según referencias históricas sería Einthoven en 1905 quien experimenta por vez primera al transmitir datos de los registros obtenidos por un galvanómetro vía telefónica (Willem Einthoven, 2019). En la década de los sesenta, se documenta un encuentro de telemedicina mediante el uso de un circuito cerrado de televisión entre el Instituto Psiquiátrico de Nebraska, en Omaha, y el Hospital Estatal de Norfolk, a 112 millas de distancia, conduciendo sesiones de terapia de grupo, y en 1967, se implementa el primer prototipo de un sistema interactivo de telemedicina que unía una estación médica en el aeropuerto de Boston y el Hospital General de Massachussets. Posteriormente, mediante videoconsulta se realiza asistencia para cirugía de corazón entre colegas de EEUU y Suiza (Bashshur et al., 2000).

En este proceso de franco desarrollo, las modalidades de interacción entre el personal de salud y sus pacientes apoyadas por las innovaciones tecnológicas se diversificaron asumiendo múltiples denominaciones; réplicas informáticas de las físicas ya existentes. Las incorporaciones más notables fueron los llamados expedientes clínicos electrónicos, (Electronic Health Records 'eHR's) en los años noventa (Institute of Medicine, 2001). Posteriormente, la receta electrónica y la agenda electrónica, que al día de hoy suman decenas de innovaciones (World Health Organization [WHO], 2006).

La International Telecommunication Union ([UIT], 2008), estudia y determina que durante el periodo 1960-2000, el "ciclo tradicional de proyectos de telemedicina" fue decepcionante, decenas, centenas y miles de pruebas piloto se desarrollaron, solo algunas de las iniciativas consiguieron sobrevivir más allá del final de su período de financiación inicial. La conclusión de su informe detalla que durante el siglo XX, menos del 10\% de los proyectos en los países en desarrollo tuvieron éxito, con un vacilante $45 \%$ después de sólo 1 año y el restante $45 \%$ al cabo de 3 años.

Desde el 2004 hay en marcha por influjo de la Comisión Europea, el denominado Plan de Salud Electrónica, que facilita con apoyo tecnológico la intercomunicación entre centros. La Union Europea crea el "Espacio Europeo de Salud en Línea" para coordinar actividades de exclusivo desarrollo virtual que facilite el intercambio de informacion, siendo este mismo 
organismo en su plan 2012-2020 el que fija como objetivo mejorar la calida de vida y potenciar el desarrollo de la denominada e-Salud (European Commission, 2012).

Para el 2005, en reunión de la Organización de Naciones Unidas, que integró a 192 países conmemorando la $58^{\mathrm{a}}$ Asamblea de la Organización Mundial de la Salud (OMS) aprobaron la resolución sobre Cibersalud (WHO, 2005). En las memorias consta el documento que define a la e-Salud como: "el uso coste-efectivo y seguro de las tecnologías de la información y comunicación en apoyo de la salud y de los ámbitos relacionados con la salud, incluyendo los servicios de atención sanitaria, vigilancia de la salud, literatura y educación, conocimiento e investigación” (WHO, 2004).

La Organización Panamericana de la Salud (OPS) desarrolló similar iniciativa en América Latina con la "Estrategia y Plan de Acción sobre e-Salud de 2012 - 2017" (Panamerican Health Organization, 2011).

Se puede afirmar que sucedió ya en el mundo entero, el tránsito de la telemedicina como herramienta de comunicación e interacción entre profesionales, a la tele salud, como insumo para la comunicación e interacción entre médicos y pacientes.

La Organización Mundial de la Salud formula definiciones precisas sobre términos a fines a la era de la salud digital (WHO, 2019):

- e-Salud: Uso de las tecnologías de la información y comunicación en apoyo de la salud $\mathrm{y}$ aspectos relacionados.

- Telemedicina: Aportar servicios de salud, donde la distancia es un factor crítico, por cualquier profesional de la salud, usando las nuevas tecnologías de la comunicación para el intercambio válido de información en el diagnóstico, tratamiento y prevención de enfermedades o lesiones, investigación y evaluación, y educación continuada de los proveedores de salud, todo con el interés de mejorar la salud de los individuos y sus comunidades.

- m-Salud: Utilización de tecnologías inalámbricas móviles en salud.

- Salud digital: Término amplio que incluye e-Salud, m-Salud, así como el uso de tecnologías avanzadas de computación (big data), genómica e inteligencia artificial.

- Tele salud: Utilización de las telecomunicaciones y de tecnología virtual en la asistencia sanitaria fuera del ámbito hospitalario o de atención tradicional.

En épocas de la pandemia, incorporar en unos sistemas y potenciar en otros el enfoque ergonómico y factores humanos (FH), permitió que pueda ser un elemento diferenciador, dinamizador y seguro en las instituciones o empresas como adyuvante en la atención y vigilancia de la salud, para el manejo o control de casos categorizados como probables, sospechosos o confirmados de COVID-19.

La OPS y la OMS en su hoja informativa No 7 "COVID-19 y el Rol de los Sistemas de Información y las Tecnologías en el Primer Nivel de Atención”, mencionan que las tele consultas son una forma segura y efectiva de evaluar casos sospechosos y guiar el diagnóstico y el tratamiento del paciente, minimizando el riesgo de transmisión de la enfermedad (2020). 
La tecnología mínima necesaria que se requiere para poder en la práctica realizar una tele consulta son, una conexión a internet y una computadora adecuada con capacidades de audio y video y dispositivos de transmisión. Se recomiendan conexiones rápidas y estables a Internet y banda ancha (ADSL, fibra óptica, cable, 4G o similar; al menos $1 \mathrm{MB} / 300 \mathrm{~kb}$ ). También es necesario saber utilizar un software específico y herramientas de conferencia, como Webex, Skype, Zoom, Elluminate, MS Teams y FaceTime.

Como soporte a este nuevo quehacer, la OPS (2020, 8 de septiembre) ha formulado una herramienta que forma parte del apoyo a las operaciones de respuesta a la pandemia, para la modalidad virtual, basada en los modelos de distintas instituciones de salud y niveles de complejidad, producto de la colaboración de instituciones y expertos especialistas en el área, incorporando seis componentes: la preparación organizacional, los procesos, el entorno digital, los recursos humanos, los normativos y el conocimiento especializado. Al final de la misma contiene una guía de orientación de los resultados que se convierten en recomendaciones para la gestión

En el marco de la pandemia, desde la OPS (2020) se elaboró un documento denominado "Recomendaciones para la Reorganización y Ampliación Progresiva de los Servicios de Salud para la Respuesta a la Pandemia de COVID-19", en el que entre múltiples apoyos organizativos, tecnológicos, humanos recomendados, cita al fortalecimiento de la atención domiciliar y remota. También se incorporan recomendaciones de orden técnico necesarias para el componente de salud laboral por posibles pacientes sospechosos o confirmados. La OPS y la OMS, emiten como documento guía el "Marco de Implementación de un Servicio de Telemedicina" (2016). De acuerdo a este, los servicios de telemedicina están perfectamente diseñados y acondicionados para ayudar a alcanzar los objetivos que se derivan de las siguientes tres prioridades:

1. La telemedicina protege activamente a los trabajadores de la salud al reducir las interacciones no agudas entre pacientes y personal de salud, lo que minimiza el riesgo de transmisión del COVID-19.

2. La tele consulta ayudará a las comunidades a proteger a las personas de alto riesgo (los ancianos y las personas con condiciones de salud concomitantes) al reducir su exposición en hospitales y centros con alta carga infecciosa potencial.

3. Los países o regiones con gran cantidad de personal y recursos de atención médica podrán ayudar a los países o regiones con acceso limitado, proporcionando personal y recursos (Chauhan, 2020).

Según registro y proyecciones de la Comisión Económica para América Latina y el Caribe ([CEPAL], 2008), el Ecuador tiene $256.370 \mathrm{~km} 2$ de territorio, distribuido en 24 provincias, con 17.510.643 habitantes, los que serán para el 2030, 19,7 millones; para el 2040, 21,6 millones; y para el 2050, 23,0 millones.

En Ecuador para el inicio de la pandemia el sistema de salud estaba constituido según el registro "Estadístico de Recursos y Actividades de Salud" (Instituto Nacional de Estadística y Censos, 2020, 1 de abril), de 4.165 establecimientos de salud a nivel nacional, de los cuales, 626 correspondían a establecimientos con internación hospitalaria y 3.539 sin internación. 
Para el 2018, el número de médicos y odontólogos fue de 29.604 y 3.805, respectivamente; con ello el país tenía 23,44 médicos y 3,12 odontólogos por cada diez mil habitantes. De los otros profesionales de la salud, habían 0,93 psicólogos, 14,54 enfermeras, 1,35 obstetrices y 10,19 auxiliares de enfermería, por cada diez mil habitantes.

Se efectuaron en 2018, 39,8 millones de consultas atendidas por un médico, psicólogo u obstetra a pacientes por una enfermedad determinada (morbilidad); el 61,07 por ciento correspondió a mujeres y el 38,93 por ciento a hombres. Para el 2020 en tiempos de pandemia, se produjo un incremento de 348 camas UCI a nivel nacional equivalente a $134 \%$, así como de los ingresos y hospitalizaciones en todo el territorio nacional.

Según el Ministerio de Salud Pública del Ecuador, a junio 2021 se confirmaron 439.000 casos de COVID-19 a nivel nacional (figura 1), de los cuales 7.348 fueron de profesionales de la salud, representando el 2 por ciento del total; de estos casos, 3.431 correspondieron a médicos, 2.182 enfermeras y enfermeros, 1.177 auxiliares de enfermería, 159 obstetrices y obstetras, 256 odontólogas y odontólogos, y 143 psicólogas y psicólogos. Por otra parte, a esa fecha, se reportaron 21.051 muertes, de las cuales, de acuerdo a cifras oficiales, 81 eran profesionales de la salud, con 74 confirmados y 7 probables (2020, 10 de septiembre).

Figura 1. Curva epidemiológica COVID-19. Febrero 2020 - mayo 2021.

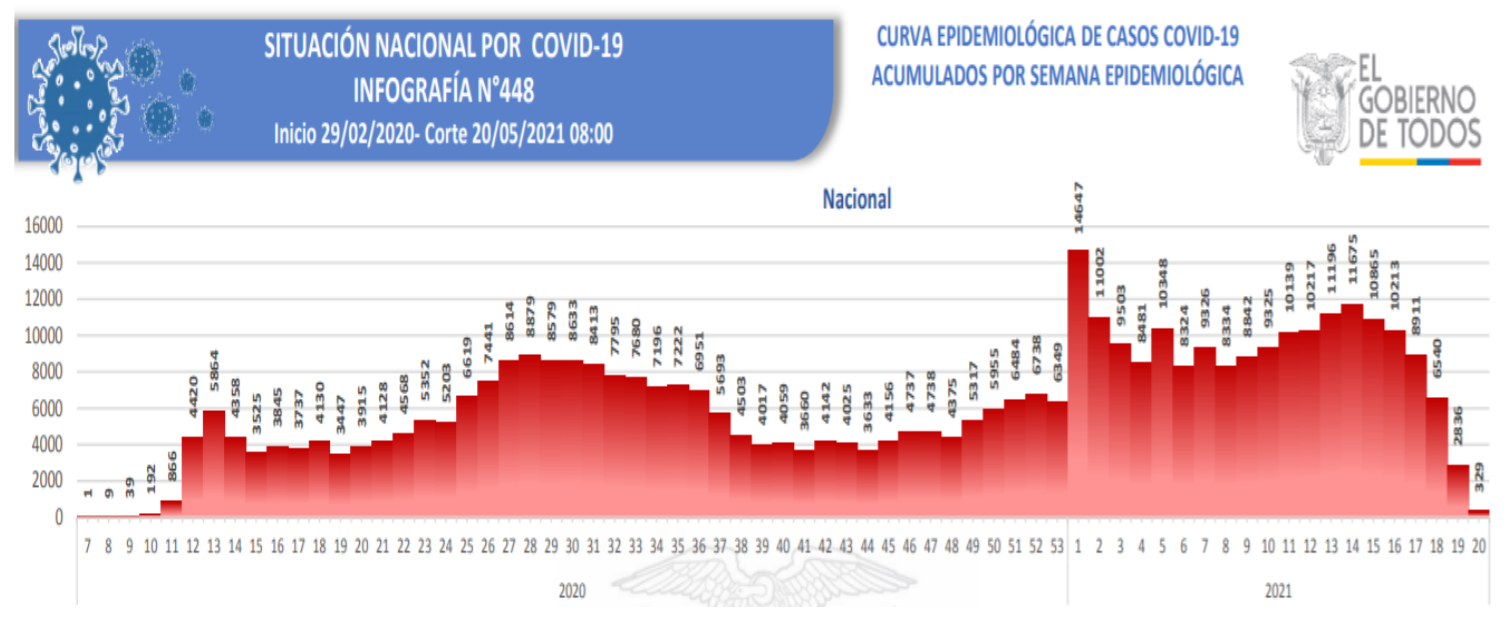

Fuente. Ministerio de Salud Pública del Ecuador (2020, 10 de septiembre).

\section{Materiales y métodos}

En el escenario de pandemia frente a la realidad de los establecimientos de salud públicos y privados tanto ambulatorios como hospitalarios, se desarrolló la presente experiencia en cinco empresas privadas que contaban con servicio médico de empresa y cuyo personal fue enviado a cuarentena obligatoria por disposición del Estado inicialmente, y luego a teletrabajo en diferentes fases y periodos de tiempo. En este personal, en distintos momentos se presentaron casos sospechosos, posibles y confirmados de COVID-19.

Inicialmente ninguna de las empresas contaba con recursos tecnológicos funcionales para vigilancia de la salud remota. Todas ellas eran heterogéneas en sus actividades, número 
de colaboradores y composición de género. Las actividades desarrolladas eran tanto administrativas como operativas, de tipo automotriz, transporte, envasado de combustible, metalmecánica y educativa, con un número de 200 colaboradores en total.

Criterios de selección

Se incluyó a todo el personal en funciones, sin limitaciones de edad, género, actividad o tiempo de servicio en cada empresa.

\section{Procedimiento}

Se asignó un profesional médico con especialidad en salud ocupacional y medicina del trabajo con subespecialidad en ergonomía, con experiencia y vinculación previa a entidades públicas y privadas, que conoce las empresas y su personal; se seleccionaron dos vías de comunicación para una aplicación efectiva:

- La primera una encuesta digital utilizando la plataforma Google Drive a la que se denominó: Encuesta Diaria de Salud ([ENDISA], ver en anexo figura A1), la que contenía 13 preguntas de fácil respuesta en las que se detallaron un listado de signos y síntomas en orden de frecuencia de presentación, desde los más comunes o menos graves a los más significativos. Se estimó en pruebas piloto que su respuesta no requería más de 2 minutos. Se envió cada día antes de entrar a la jornada de trabajo vía WhatsApp al móvil personal de los trabajadores. La plataforma brindó la posibilidad de, en tiempo real, enviar notificaciones del ingreso de respuestas y poder revisar las estadísticas totales, así como el detalle de cada una de las encuestas al médico asignado, facilitando localizar a un potencial caso sintomático o dar seguimiento a un confirmado en aislamiento preventivo obligatorio (APO).

- Como segundo elemento del sistema, mediante una plataforma específica para tele salud, se creó la historia clínica digital de todos los vigilados y en cuanto se requería, se agendaba una video consulta, la misma que notificaba automáticamente al colaborador mediante un mensaje de texto al mail y al móvil por WhatsApp precisando datos como: el profesional que atenderá, la hora y día. Antes de iniciar la video consulta, la plataforma emitía una nueva notificación como recordatorio previo y un enlace para acceder a la cita programada, en audio y video.

Realizada la valoración por la video consulta se definía, según el estado del paciente, el paso a seguir, que podía ser:

1. Referir a estudio de laboratorio.

2. Aislamiento con vigilancia diaria.

3. Asistencia a un centro médico de referencia predeterminado para valoración integral.

El ciclo de vigilancia se grafica en la figura 2. 
Figura 2. Ciclo dinámico para vigilancia por medios virtuales.

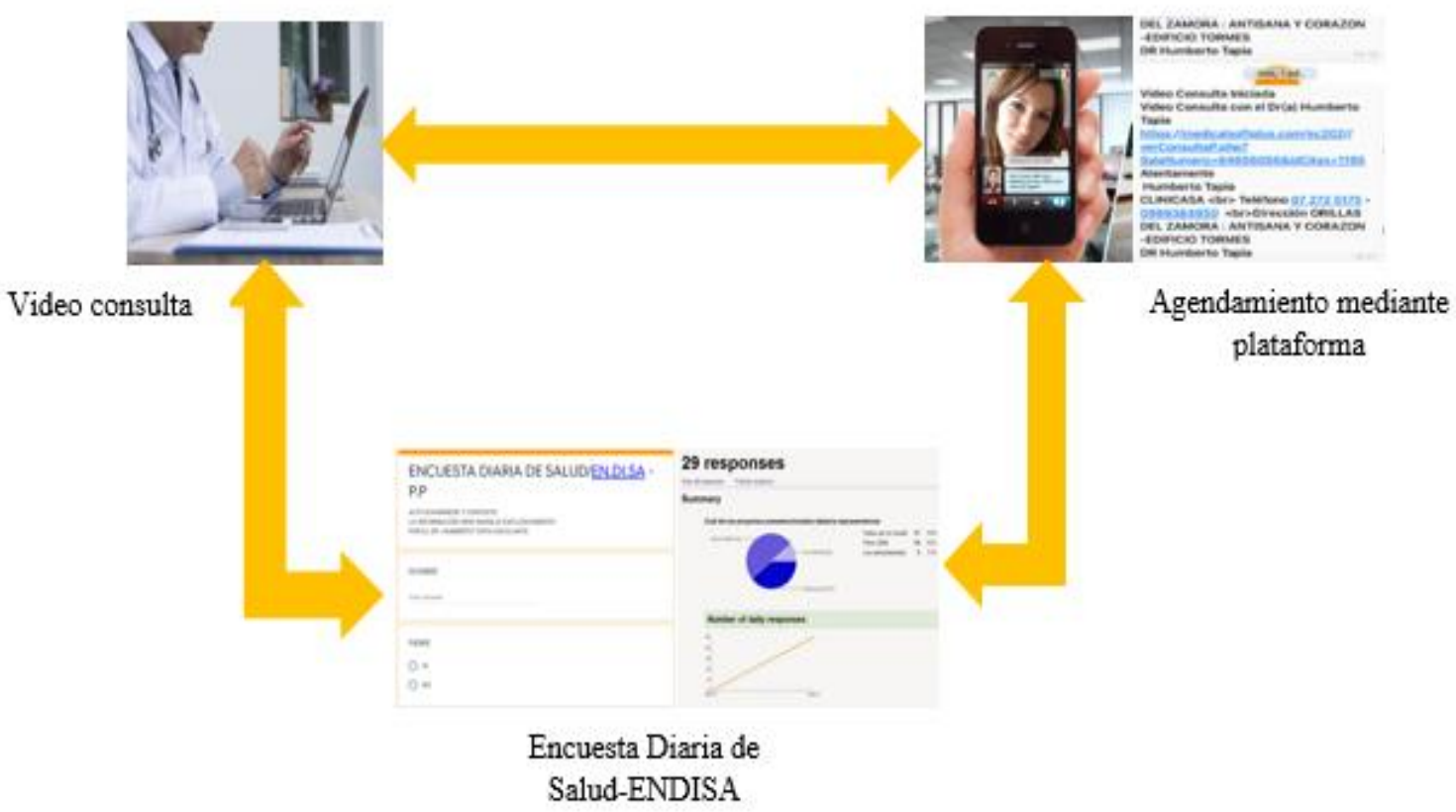

Tabla 1. Caracterización de los trabajadores vigilados en pandemia.

\begin{tabular}{|c|c|c|c|c|}
\hline $\begin{array}{c}\text { Actividad } \\
\text { empresa }\end{array}$ & $\mathbf{N}^{\circ}$ colaboradores & Género & $\begin{array}{c}\text { Actividades } \\
\text { administrativas }\end{array}$ & $\begin{array}{c}\text { Actividades } \\
\text { operativas }\end{array}$ \\
\hline Automotriz & 20 & $\begin{array}{c}50 \% \mathrm{M} \\
50 \% \mathrm{~F}\end{array}$ & $40 \%$ & $60 \%$ \\
\hline Transporte & 20 & $100 \% \mathrm{M}$ & $15 \%$ & $75 \%$ \\
\hline $\begin{array}{c}\text { Envasado de } \\
\text { combustible }\end{array}$ & 80 & $\begin{array}{c}50 \% \mathrm{M} \\
50 \% \mathrm{~F}\end{array}$ & $50 \%$ & $50 \%$ \\
\hline Metalmecánica & 40 & $\begin{array}{c}95 \% \mathrm{M} \\
5 \% \mathrm{~F}\end{array}$ & $15 \%$ & $85 \%$ \\
\hline Educativa & 40 & $\begin{array}{c}85 \% \mathrm{~F} \\
15 \% \mathrm{M}\end{array}$ & $10 \%$ & $90 \%$ \\
\hline
\end{tabular}

Nota. M: masculino, F: femenino.

\section{Resultados y discusión}

Se procesó cada día de los 200 colaboradores de las empresas, las 13 respuestas a la encuesta ENDISA totalizando 2.600 respuestas diarias, durante los meses de julio a diciembre de 2020; 10\% de los colaboradores de las empresas fueron diagnosticados en momentos diferentes por laboratorio mediante técnica de PCR por hisopado nasal como COVID-19 positivo, con afectación de su entorno familiar (uno o más de sus familiares directos con los que conviven fueron positivos); el $50 \%$ correspondieron a conductores de vehículos de transporte, el $30 \%$ a docentes, el 10\% a operativos de tipo automotriz y metalmecánica, y el $10 \%$ a administrativos.

Por la vigilancia se identificaron señales de alerta temprana o fase de la enfermedad en la que se encontraba. El 1\% ameritó consulta presencial para re-evaluación y el o,5\% ingresó 
a unidad de cuidados intermedios. No se produjo ninguna letalidad en el grupo de los vigilados.

A los casos diagnosticados como positivos se les realizó vigilancia mediante controles diarios por la ENDISA y video consultas para valoración durante 15 días continuos hasta que la fase aguda se controlara, remitieran los signos y síntomas o se reunieran criterios para el alta clínica. En el domicilio se registró la tensión arterial, frecuencia cardiaca, temperatura y saturación de oxígeno. Terminada esta fase se descontinuaron los controles diarios y se mantuvieron a demanda espontánea dependiendo de novedades de los pacientes que ameritaban alguna consulta y orientación. Este procedimiento se extendió a las familias que solicitaron el apoyo.

Los pacientes que ameritaron valoración física fueron aquellos que por los datos reportados en su monitoreo diario tenían tendencia a salir de los rangos normales, justificándose una valoración integral, exámenes complementarios, y en un caso, internamiento por 7 días en cuidados intermedios, que posteriormente fue dado de alta a su domicilio.

El 90\% de los colaboradores no infectados, fueron vigilados por contactos o actividades riesgosas como atención al público y/o viajes; de todos se documentó su evolución diaria que fue satisfactoria en cuanto a las patologías de temporada como a las preexistentes.

Tabla 2. Número de video consultas y encuestas realizados en línea durante vigilancia.

\begin{tabular}{|c|c|c|}
\hline Metodología & Pacientes & Total semestral \\
\hline Video consultas & Positivos COVID-19/APO & 300 \\
\hline Encuesta ENDISA & Total población vigilada & 36.000 \\
\hline
\end{tabular}

\section{Discusión}

Dado que el objetivo de la ergonomía es "promover la salud y el bienestar, reducir los accidentes y mejorar la productividad" (Apud \& Meyer, 2003, pp. 15-20), y desde el punto de vista de los factores humanos, la "clave para mejorar los sistemas evitando así accidentes, eventos adversos y complicaciones que puedan afectar directamente la seguridad de los pacientes y de los trabajadores de salud" (Hignett et al., 2013, pp. 56), se convierte en la "herramienta indispensable para mejorar o adecuar cualquier contexto de prestación de salud a los requerimientos que el momento exija, mejorando el bienestar de los usuarios, así como optimizar el desempeño de los sistemas" (Dul et al., 2012, pp. 55).

Las características descritas evidencian que en tiempos considerados normales la ergonomía y FH son fundamentales para el quehacer diario, pero en épocas atípicas como las actuales se convierten en indispensables. Según la OPS (s.f.) en los países de las Américas $(29,3 \%)$ los pobladores no buscaron atención en salud cuando lo necesitaron debido a múltiples barreras de acceso; el 17,2\% lo atribuyó a barreras organizativas (ej. largos tiempos de espera, horas de atención inadecuadas, requisitos administrativos engorrosos), el 15,1\% de los casos por barreras financieras, el 8,4\% de los casos por disponibilidad inadecuada de recursos (ej. falta personal de salud, medicamentos e insumos), y el 5,4\% de los casos por barreras geográficas. La opción seleccionada, motivo de esta experiencia, para la atención y vigilancia de la salud, en este tiempo subsana algunas de las limitaciones del sistema de salud 
o de la atención convencional como: la accesibilidad, la cobertura, gratuidad, la confidencialidad, etc., y permite interactuar casi sin restricciones entre quienes requieren y ofrecen el servicio.

El mayor aporte de incorporar el componente ergonómico y de FH en este modelo de atención radica en readecuar el sistema vigente institucional o empresarial, rescatando como protagonistas de esta historia a la "persona paciente" y a la "persona profesional de la salud", los dos con realidades paralelas que requieren encontrarse en el marco de la planificación y ejecución de una forma alternativa de interactuar basados en sus necesidades y formación respectivamente.

En países de la región se ajustaron o emitieron normativas específicas para la aplicación de tele-vigilancia en este contexto. En Perú, según cita Vadillo-Vila (2020, 30 de abril), en el Ministerio de Salud solo entre el 13 al 25 de abril del 2020 se registraron 4.310 solicitudes de atención a distancia.

En Chile que han desarrollado acciones ya desde el 2005 hasta diciembre al 2017, se han realizado más de 6 millones de tele asistencias (Ministerio de Salud de Chile, 2018).

Según el Ministerio de Salud Pública del Ecuador (2020, 10 de septiembre), solo en pandemia se realizaron, 2.095.419 llamadas al 171 relacionadas a COVID-19; 481.258 seguimientos telefónicos a pacientes con diagnóstico confirmado, y 138.773 tele consultas a ciudadanos atendidos a través de la aplicación SALUDEC por un médico del 171.

Hay múltiples ejemplos ya en la región y en el mundo de estudios que se están desarrollando sobre la satisfacción de los usuarios. La Asociación Americana de Gastroenterología mediante encuesta virtual a más de 2.000 personas, mostró que el $66 \%$ estaba de acuerdo con usar la tele salud, con un alto nivel de interés en todos los grupos de edad, incluido el $74 \%$ de encuestados de 18 a 44 años, y el $52 \%$ de 65 años y mayores. En total, el 25 \% informó que estaría dispuesto a cambiar a su médico de atención primaria por uno que ofreciera tele salud; pero solo el $8 \%$ reportó una experiencia real con una visita de video con un médico (Lee \& Kim, 2020).

En la presente experiencia ninguno de los atendidos reportó por vía formal o informal reparo por algún grado de insatisfacción en la atención. Con una baja inversión y apoyados en la tecnología existente se logró atender y vigilar a grupos de colaboradores cautivos por las actuales circunstancias sanitarias, lo que resultó altamente efectivo en la patología infecciosa de temporada, como en afecciones comunes preexistentes o nuevas que ameritaban orientación o manejo.

Sin embargo, en el desarrollo se presentaron limitaciones iniciales de asimilación de los directivos sobre las bondades y los beneficios a obtenerse, y algo de desconfianza previa por los colaboradores acostumbrados a la atención y relación personal convencional con su médico, subsanado todo ello con el tiempo y los resultados obtenidos. 


\section{Conclusiones}

Las actividades de vigilancia y atención de la salud pueden beneficiarse de incorporar a su sistema de gestión, entre otras opciones, de la vía remota, apoyada con la tecnología actual, para la generación de historia clínica digital, video consulta, receta electrónica y monitoreo diario, en patologías altamente contagiosas y en entornos de cuarentena o aislamiento, pero también en patologías no trasmisibles, crónicas, degenerativas, catastróficas, etc.

Se constituye una forma idónea de interactuar con las personas, brindando acompañamiento, una visión real diaria de la evolución y la expectativa de poder responder a tiempo en las contingencias, independientemente del lugar, hora y el día en el que se requiera. Un solo profesional puede atender a un número importante de personas en tiempo real, en diferentes localidades, optimizando tiempos injustificados de traslados, turnos, recuperación de expedientes, etc., lo que no se podría realizar bajo la modalidad convencional de atención.

En la pandemia actual la medida de bioseguridad más efectiva ha sido el distanciamiento físico, evitando contactos y contagios entre las personas, más aun con el personal de salud. La modalidad de atención remota, si bien ha ganado presencia por sus resultados, aún experimenta limitaciones de orden tecnológico, técnico y económico, que serán progresivamente superadas para potenciar su uso en niveles de diferente complejidad, para lo cual, es fundamental considerar el enfoque ergonómico y de FH. 


\section{Referencias}

Apud, E., \& Meyer, F. (2003). La importancia de la ergonomía para los profesionales de la salud. Ciencia y enfermería, 9(1), 15-20. http://dx.doi.org/10.4067/So717-95532003000100003

Bashshur, R. L., Reardon, T. G., \& Shannon, G. W. (2000). Telemedicine: A new health care delivery system. Annual Review of Public Health, 21, 613-637. https://doi.org/10.1146/annurev.publhealth.21.1.613

Chauhan, V., Galwankar, S., Arquilla, B., Garg, M., Di Somma, S., El-Menyar, A., Krishnan, V., Gerber, J., Holland, R., \& Stawicki, S. P. (2020). Novel coronavirus (COVID-19): Leveraging telemedicine to optimize care while minimizing exposures and viral transmission. Journal of $\begin{array}{llll}\text { Emergencies, } \quad \text { Trauma, } & \text { and }\end{array}$ https://dx.doi.org/10.4103\%2FJETS.JETS 3220

Comisión Económica para América Latina y el Caribe. (2008). Transformaciones demográficas y su influencia en el desarrollo en América Latina y el Caribe. https://www.cepal.org/es/publicaciones/2894-transformaciones-demograficas-suinfluencia-desarrollo-america-latina-caribe

Dul, J., Bruder, R., Buckle, P., Carayon, P., Falzon, P., Marras, W. S., Wilson, J. R., \& van der Doelen, B. (2012). A strategy for human factors/ergonomics: Developing the discipline and profession. Ergonomics. 55(4), 377-395. https://doi.org/10.1080/00140139.2012.661087

European Commission. (2012). Communication from the commission of the european parliament, the council, the european economic and social committee, and the committee of the regions ehealth action plan 2012-2020. Innovative healthcare for the 21st century. https://eur-lex.europa.eu/legal-content/EN/ALL/?uri=CELEX\%3A52012DCo736

Hignett, S., Carayon, P., Buckle, P., \& Catchpole, K. (2013). State of science: Human factors and ergonomics in healthcare. Ergonomics, 56(10), 1491-1503. https://doi.org/10.1080/00140139.2013.822932

Institute of Medicine. (2001). Crossing the quality chasm: A new health system for the 21st century. The National Academies Press. https://doi.org/10.17226/10027

Instituto Nacional de Estadística y Censos. (2020, 1 de abril). Estadísticas de Recursos y Actividades de Salud. Gobierno de la República del Ecuador. https://www.ecuadorencifras.gob.ec/inec-difunde-estadisticas-de-recursos-y-actividadesde-salud-2018/

International Telecommunication Union. (2008). Implementing e-Health in developing countries. Guidance and principles. https://www.itu.int/ITU-D/cyb/app/docs/eHealth prefinal 15092008.PDF

Lee, T., \& Kim, L. (2020). Telemedicine in gastroenterology: A value-added service for patients. $\begin{array}{llll}\text { Clinical gastroenterology and hepatology, 18(3), 530-533. } & \text {. }\end{array}$ https://doi.org/10.1016/j.cgh.2019.12.005

Ministerio de Salud de Chile (2018). Programa nacional de telesalud. En el contexto de Redes Integradas de Servicios de Salud. https://www.minsal.cl/wpcontent/uploads/2018/03/Programa-Nacional-de-Telesalud.pdf

Ministerio de Salud Pública del Ecuador. (2020, 10 de septiembre). El MSP informa: Situación coronavirus COVID-19. https://www.salud.gob.ec/el-ministerio-de-salud-publica-delecuador-msp-informa-situacion-coronavirus/

Organización Panamericana de la Salud. (2020). Recomendaciones para la Reorganización y Ampliación Progresiva de los Servicios de Salud para la Respuesta a la Pandemia de 
COVID-19. https://www.paho.org/es/documentos/recomendaciones-para-reorganizacionampliacion-progresiva-servicios-salud-para-respuesta

Organización Panamericana de la Salud. (2020, 8 de septiembre). Nueva herramienta busca medir el nivel de madurez de instituciones de salud para ofrecer telemedicina en tiempos de pandemia. https://www.paho.org/es/noticias/8-9-2020-nueva-herramienta-busca-medirnivel-madurez-instituciones-salud-para-ofrecer

Organización Panamericana de la Salud. (s.f.). Salud universal. Consultado el 23 de septiembre de 2020. https://www.paho.org/es/temas/salud-universal

Organización Panamericana de la Salud \& Organización Mundial de la Salud. (2016). Marco de implementación de un servicio de telemedicina. http://iris.paho.org/xmlui/bitstream/handle/123456789/28413/9789275319031 spa.pdf?se quence $=6$

Organización Panamericana de la Salud \& Organización Mundial de la Salud. (2020). COVID-19 y el rol de los sistemas de información y las tecnologías en el primer nivel de atención. https://iris.paho.org/bitstream/handle/10665.2/52205/COVID19FactsheetPNA\%20 spa.pdf?sequence=14

Panamerican Health Organization (2011). Strategy and plan of action on eHealth. http://www.paho.org/hq/index.php?option=com content\&view=article\&id=5723\&Itemid= 4139\&lang=es

Vadillo-Vila, J. (2020, 30 de abril). 4.310 solicitudes de telemedicina recibió en dos semanas el Minsa por aplicativo. Diario El Peruano. https://elperuano.pe/noticia/95193-4310solicitudes-de-telemedicina-recibio-en-dos-semanas-el-minsa-por-aplicativo

Willem Einthoven: As part of our ongoing series of Nobel Prize winners that have contributed to cardiovascular medicine, Mark Nicholls focuses on the work of Willem Einthoven who was awarded the 1924 Nobel Prize in Physiology or Medicine for his discovery of the mechanism of the electrocardiogram (2019). European Heart Journal, 4O(37), 3075-3078. https://doi.org/10.1093/eurheartj/ehz663

World Health Organization. (2004). eHealth. Report by the Secretariat. https://apps.who.int/iris/handle/10665/20235

World Health Organization. (2005). Fifty-eighth world health assembly. https://apps.who.int/gb/ebwha/pdf files/WHA58-REC1/english/A58 2005 REC1-en.pdf

World Health Organization. (2006). Electronic health records. Manual for developing countries. https://apps.who.int/iris/bitstream/handle/10665/207504/9290612177 eng.pdf

World Health Organization. (2019). Guideline: Recommendations on digital interventions for health system strengthening. https://www.who.int/reproductivehealth/publications/digitalinterventions-health-system-strengthening/en/ 


\section{Anexo}

Figura A1. ENDISA.

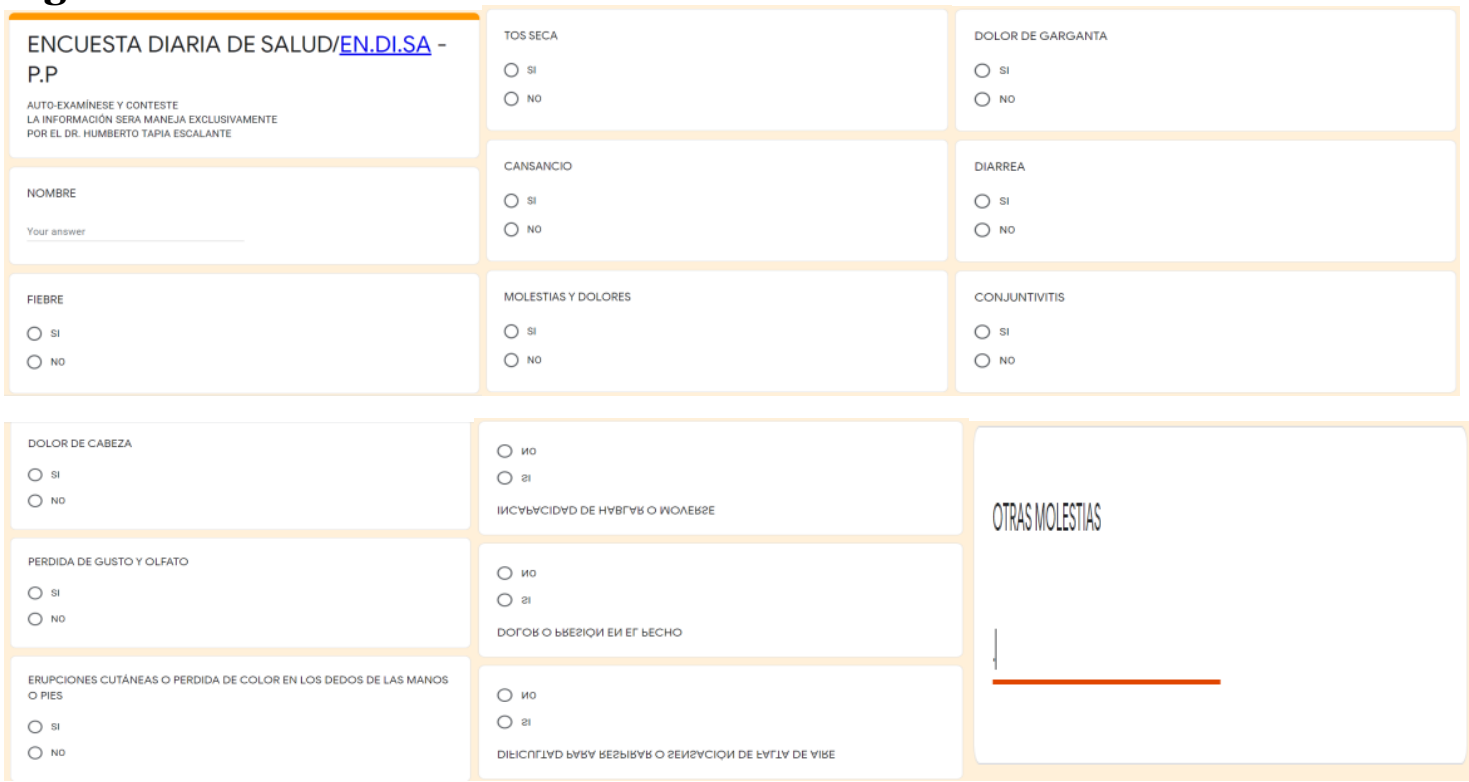

